\title{
INVESTIGACION
}

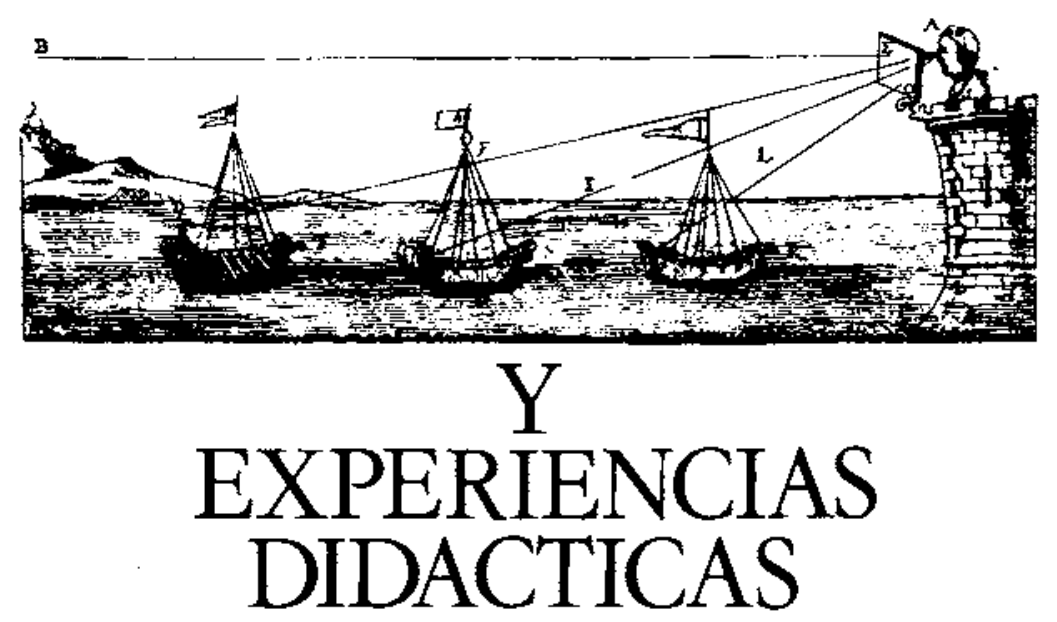

\section{EL MODELO DE AUSUBEL EN LA DIDÁCTICA DE LA FÍSICA: UNA APROXIMACIÓN EXPERIMENTAL AL PROCESO DE E/A DE CONTENIDOS QUE PRESENTAN CONSTRUCTOS POCO ELABORADOS POR LOS APRENDICES}

GÓMEZ, S. ${ }^{1}$, LATORRE, A. ${ }^{2}$ y SANJOSÉ, V. ${ }^{3}$

${ }^{\prime}$ Departament de Física Aplicada. Universitat de València.

${ }^{2}$ Departament de Psicologia Evolutiva i de l'Educació. Universitat de València.

${ }^{3}$ Departament de Didàctica de les Ciències Experimentals. Universitat de València.

\section{SUMMARY}

This paper shows the results of a research on physics education about «Magnetism» in secondary school. We investigate a constructivist approach to instructional design based upon Ausubel's model in the treatment of subjects on which pupils have poor conceptual representations and alternative frameworks. The first results with respect to inmediate and lasting learning, are shown.

\section{INTRODUCCIÓN}

Desde la aparición de la teoría del aprendizaje significativo (Ausubel 1976, Novak 1979, 1986) hasta hoy, la investigación teórica $-\mathrm{y}$, en menor medida, la experimental-en didáctica de las ciencias ha avanzado consi- 
derablemente los puntos de partida que están convergiendo en lo que se ha dado en Ilamar constructivismo (Coll 1987, Langford 1989, Edwards y Mercer 1988, Novak 1987). Esta evolución, que ha traído consigo la defensa de un planteamiento en la acción crítico con las metodologías fundamentalmente verbales (Gil 1983, 1986), no se corresponde con la práctica habitual en docencia en donde el libro de texto tradicional y el aprendizaje por recepción siguen siendo dominantes, habida cuenta de las condiciones en las que trabajan la mayoría de los profesionales de la enseñanza.

Asumiendo los planteamientos constructivistas, pensamos que podemos formular aún preguntas básicas: ¿Puede lograrse una mejora sensible (y medible) en el aprendizaje significativo de las ciencias con diseños adecuados a partir de la teoría de Ausubel? ¿En qué aspecto o variable de la Situación Educativa podemos esperar mayores ventajas del uso de la teoría? ¿Qué estrategias pueden resultar más efectivas a la hora de potenciar tanto la construcción activa de significados en los aprendices como la reflexión crítica y la investigación en el aula por parte del profesor?

Por ello, nuestro propósito se centra en la investigación -de campo y en condiciones reales a todos los efectosde la efectividad que puede tener un planteamiento ausubeliano bien estructurado. Al mismo tiempo, pretendemos adentrarnos en los caminos que llevan a los alumnos a unas u otras construcciones de conceptos y en la dependencia que ello tiene con las variables de la situación educativa (Latorre 1990).

Este modelo sólo ha demostrado limitaciones en los primeros niveles de escolaridad (Fortes 1991), y en algunos núcleos conceptuales en enseñanza secundaria como mecánica, calor u óptica, con presencia de constructos personales sólidos (Kelly 1955). En estos últimos casos, parece probado que se necesita la activación de mecanismos metodológicos profundos, asociados a objetivos de tipo cambio conceptual (Posner et al. 1982, GiI et al. 1991).

Sin embargo, ¿sucede lo mismo en el caso del tratamiento de contenidos más alejados de las experiencias personales o que necesitan de un nivel de abstracción superior? En este sentido, creemos que el tema «Magnetismo» puede ser apropiado para investigar la efectividad de un diseño de instrucción llevado a la práctica a través de una metodología ausubeliana.

\section{OBJETIVOS DE LA INVESTIGACIÓN}

Diseñamos una experiencia piloto fundamentada en un método de valoración del aprendizaje basado en el diseño experimental pretest-postest. A partir de los resultados de la misma, hemos extraído la información necesaria para depurar y perfilar los instrumentos de trabajo así como el método en su conjunto, con el fin de aplicarlos de forma sistemática a otras investigaciones futuras.
Suponemos que un diseño de instrucción que parta de conceptos inclusores presentes en la estructura cognitiva de la mayoría de los aprendices-principio básico de esta teoría, contrastado experimentalmente en diversas investigaciones (Gilbert y Waits 1983, Saltiel y Viennot 1985, Driver y Erickson, 1983, Clough y Driver 1986)y, correspondientemente, basadoen organizadores anclados a los mismos producirá aprendizaje significativo en los alumnos, tanto a corto como a largo plazo. Por otra parte, puede lograr que aquellos alumnos que revelen un bajo nivel de dominio del contenido en el pretest alcancen tras la SEA niveles de dominio similares, por término medio, a los obtenidos por aquellos otros mejor preparados inicialmente. El establecimiento de un punto de partida accesibie para todos los alumnos, debe garantizar un avance sistemático en el dominio de los contenidos por parte de la mayoría de los estudiantes.

Por tanto, las hipótesis de trabajo a contrastar en esta investigación son las que siguen a continuación:

\section{Hipótesis 1 (Principal)}

«En el tratamiento de contenidos cuyas representaciones conceptuales previas en la mente de los aprendices presenten un grado de estructuración bajo, una instrucción basada en la teoría del aprendizaje de Ausubel debe producir diferencias significativas entre los niveles de conocimiento previo y posterior a la situación de enseñanza/aprendizaje.»

\section{Hipótesis subsidiarias}

\section{Hipótesis 1.1}

«Si se adecúa la exigencia conceptual a objetivos básicos, la elección de organizadores previos basados en conceptos inclusores de amplia presencia en la estructura conceptual de los aprendices, debe conducir, por término medio, a un efecto de homogeneización en el dominio: el rendimiento en alumnos con menor dominio inicial del contenido, será similar al de aquéllos que demuestran un dominio inicial superior.»

Es decir, las diferencias en el rendimiento de alumnos mejor preparados inicialmente respecto a los peor preparados, sólo serán perceptibles, en todo caso, en aquellos núcleos conceptuales que supongan niveles de dominio profundo del contenido y que necesiten tratamiento específico en la SEA.

\section{Hipotesis 1.2}

«Una instrucción basada en la teoría del aprendizaje de Ausubel debe retener en la «memoria a largo plazo» las estructuras de conocimiento construidas e incorporadas a la misma durante la situación de enseñanza/aprendizaje.» 


\section{MÉTODO}

Dadas las características de una investigación piloto, vimos la conveniencia de fijar el mayor número de variables posible: un centro, un nivel, un profesor y una unidad temática.

\section{Población}

La investigación fue llevada a cabo con un total de 90 alumnos, en un centro docente privado de bachillerato, homologado, situado en la comarca valenciana del Camp de Morvedre.

Los tres grupos con los que se trabajó correspondían al nivel de $3^{\circ}$ de BUP. Dichos grupos presentaban un rendimiento medio general, y poseían las características habituales de la mayoría de cursos de enseñanza secundaria: una gran heterogeneidad respecto a capacidades intelectuales, conducta, rendimiento académico, hábitos de estudio y motivación por la asignatura.

El profesor que participó en la SEA fue el titular que impartía la asignatura, con una experiencia docente, en este nivel, de doce años.

\section{Contenido}

El tema elegido fue «magnetismo», adaptado a las exigencias y limitaciones del programa oficial actualmente. En cuanto a los objetivos a cubrir, nos centramos en los que consideramos como el conjunto mínimo que presenta coherencia interna.

Los objetivos que establecimos como mínimos supuestamente obtenidos en cursos anteriores, así como los mínimos y los de profundización en el nivel de $3^{\circ}$ đe BUP que se pretendía que fuesen alcanzados durante la SEA figuran en el anexo 1.

\section{Diseño de la investigación}

Aplicamos el diseño experimental haciendo pasar a los grupos por las situaciones de pretest, SEA y postest. EI tiempo total dedicado en la SEA fue de 6 sesiones de 50 minutos.

La contrastación de la hipótesis 1 tendrá lugar miđiendo incrementos de aprendizaje entre los tests de conocimientos previo y posterior a la SEA.

Para realizar un análisis diferencial del rendimiento en función de la situación de partida (en relación con la hipotesis 1.1), clasificamos las pruebas cumplimentadas por los alumnos formando grupos en función de los resultados del test previo. El estudio comparado del rendimiento de estos grupos en el test posterior puede permitir la contrastación de dicha hipótesis.

La confrontación đe la hipótesis 1.2 tenđría lugar comparando los resultados de los tests posterior y de «me- moria a largo plazon. Este último fue cumplimentado por los alumnos dos semanas después de haber finalizado la SEA.

Para elaborar los test de conocimientos, clasificamos el conjunto total de objetivos en siete apartados (Anexo I). De cada apartado seleccionamos un objetivo, y a éste le hicimos corresponder un concepto cuyo dominio debería ser evaluado en un ítem del test correspondiente. El tipo de ejercicios a resolver corresponde a cuestiones de respuesta múltiple (4) de las cuales sólo una es correcta. Las tres posibilidades incorrectas corresponden a soluciones determinadas a partir de planteamientos basados en concepciones o asociaciones erróneas en relación con la situación problema, algunas de las cuales elaboramos a partir de la investigación sobre errores conceptuales (Cubero 1989, Driver, Guesne y Tiberghien 1989, Osborne y Gilbert 1980, Posner 1982, Strike y Posner 1982). Ello debe permitir entender qué tipo de error se comete e investigar su origen.

Al elaborar el test previo tuvimos en cuenta los conceptos sobre el tema a desarrollar supuestamente aprendidos por el alumno en cursos anteriores. Consta de siete ítems. A priori, suponemos que habrá algunos de éstos ítems que, casi con toda seguridad, el alumno no sabrá responder correctamente. De hecho, a algunos de ellos no les corresponde ningún objetivo mínimo de los que hemos establecido para cursos anteriores. Sin embargo decidimos incluirlos para tener una referencia del nivel inicial presentado por los alumnos, y poder comparar éste con el nivel final alcanzado.

En cambio, diseñamos el test posterior en base al conocimiento que debería ser adquirido por el alumno durante la SEA. Éste consta de 12 ítems. Los siete primeros están referidos a siete objetivos mínimos de $3^{\circ}$ de BUP, y los cinco restantes a objetivos de profundización. Cada uno de los siete ítems referidos a objetivos minimos del test posterior mide el dominio del mismo objetivo del test previo, pero con un nivel de exigencia mayor.

En el anexo II se muestran, a modo de ejemplos, los dos primeros ítems de los test previo y posterior.

La SEA fue preparada estableciendo como organizador previo al tema en estudio la siguiente proposición: «Los campos magnéticos están creados por corrientes eléctricas.»

Esta proposición fue elegida a priori para estructurar el contenido como organizador debido a su simplicidad de enunciado, su poder explicativo -ya que establece el origen de la entidad en estudio-, y la facilidad con que puede incorporarse a la estructura conceptual de los alumnos en este nivel, al precisar un número reducido de conocimientos básicos previos.

El hecho de que el $97 \%$ de los alumnos respondieran correctamente al ftem 6 en el test previo (ver Resultados e Interpretación) orientó nuestra elección de inclusores para el establecimiento del organizador del tema. Este ítem medía la comprensión de interacciones entre imanes, lo cual fue tomado, precisamente, como subsuntor 
a partir del cual acceder por asociación y supraordinación al origen del campo magnético -las corrientes eléctricas-, idea designada por nosotros como organizador del tema. Para ello sólo se precisa conocer con anterioridad el concepto de corriente eléctrica (movimiento de los electrones por el interior de un conductor) y rudimentos del modelo atómico planetario (según el cual la materia estaría constituida por infinidad de espiras microscópicas de corriente en los átomos -espín y órbitas electrónicas-). El siguiente esquema asociativo de proposiciones muestra cómo procedemos en el diseño instruccional para establecer el organizador del tema a partir de los inclusores encontrados.

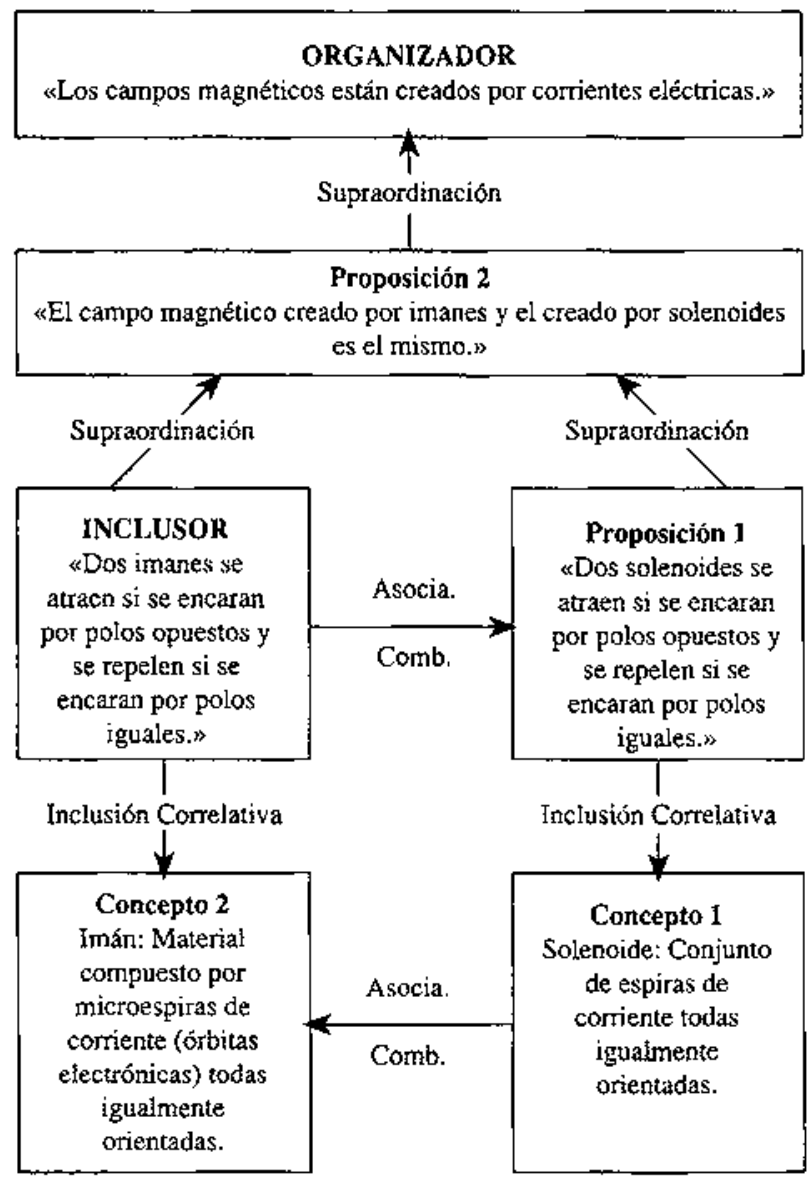

Nuestro organizador encabeza un mapa conceptual (Novak y Gowin 1988) que fue confeccionado en base al orden temporal establecido para el desarrollo de los objetivos del tema, seleccionando aquellos conceptos de más relevancia en cada uno de ellos (Anexo III).

Este mapa ha servido de base para el tratamiento jerárquico y ordenado del contenido, ya que la distribución espacial de los conceptos, junto con el sentido de sus conexiones, determinan relaciones de subordinación, supraordinación o asociación, combinatoria entre ellos. Esto permite estructurar los respectivos diseños instruccionales con arreglo a los correspondientes procesos psicológicos de deducción, inducción o asociación, que facilitan la construcción personal del aprendiz (Osborne y Wittrock 1983), necesaria para lograr un aprendizaje significativo.

Para ello hemos configurado un esquema asociativo de proposiciones de cada sesión, mediante el cual queda de manifiesto el tipo de relación psicológica que se pretende establecer entre los contenidos tratados durante la misma El expuesto anterionnente -por el cual se establece el organizador del tema-conesponde al de la primera sesión.

Por otra parte, el diseño instruccional de cada sesión contempla qué recursos y actividades didácticas deben ser dispuestos como apoyo a la exposición del tema, y en qué momentos conviene utilizarlos: Se preparó material didáctico básicamente de dos tipos: láminas de retroproyección, confeccionadas por los propios investigadores, y material experimental.

Preparamos además un bloque de ejercicios para cada sesión con el fin de facilitar a los alumnos la incorporación significativa a la MLP de los nuevos conocimientos. Los propósitos que perseguimos con estas actividades son los siguientes: confirmación, mediante ejercicios de refuerzo; generalización, mediante cuestiones que favorezcan el transfer y clasificación, mediante actividades de diferenciación progresiva. Estos bloques de actividades presentaban ejercicios de diferentes grados de dificultad, de manera que fueran útiles tanto a alumnos con un nivel alto de conocimientos previos sobre el tema como a aquéllos que presentaran inicialmente un nivel inferior.

\section{RESULTADOS E INTERPRETACIÓN}

EI diagrama de barras del gráfico 1 muestra los resultados de los tests -previo y posterior- para cada uno de los 7 objetivos mínimos medidos por las citadas pruebas. Para un objetivo determinado (eje horizontal), el porcentaje de alumnos que han resuelto correctamente el correspondiente ítem del test previo viene determinado por la altura de la barra negra. A su lado, la barra blanca nos informa sobre el porcentaje de alumnos que respondieron acertadamente el respectivo ítem del test posterior.

Gráfico l

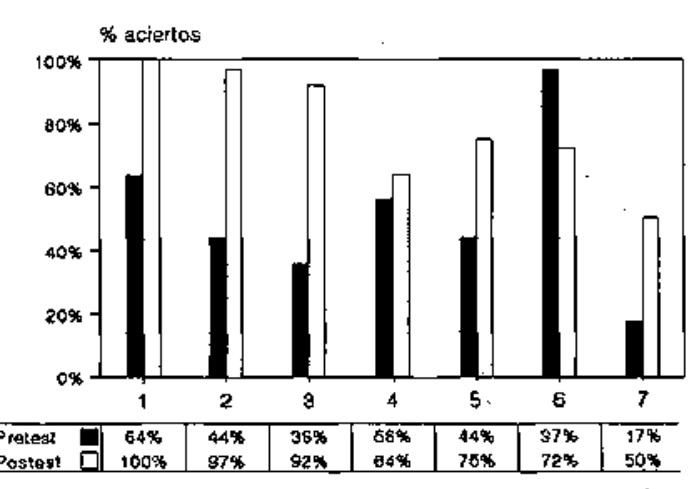

ENSEÑANZA DE LAS CIENCIAS, 1993, 1 I (3) 
Los cálculos realizados mediante el procedimiento estadístico ANOVA arrojan diferencias significativas entre ambos tests, presentando el coeficiente de Scheffe F-test un valor de 5.361 , significativo al $95 \%$. El valor medio en cada test de los referidos porcentajes y el incremento entre ambos se muestran en el cuadro l:

\section{Cuadro I}

Valor medio de ítems acertados en el test previo

Valor medio de ítems (1-7) acertados en el test posterior

Incremento absoluto

Incremento relativo
Un incremento relativo (promedio) del $53 \%$, teniendo en cuenta el mayor nivel de exigencia del test posterior respecto al test previo, nos permite concluir que durante la SEA no sólo se produjo una recuperación de bastantes conceptos olvidados -o no adquiridos anteriormente-, sino también una profundización en los mismos, hasta un $78 \%$ del dominio total del contenido programado. La hipótesis 1 queđa, en nuestra opinión, suficientemente probada.

Respecto al análisis de los ítems propuestos, como puede apreciarse en el gráfico 1 , se produjo crecimiento neto en el aprendizaje en todos los objetivos excepto en el 6, el cual presenta recesión, bajando del $97 \%$ al $72 \%$ : el nivel de exigencia había aumentado considerablemente en el test posterior respecto al previo, ya que en éste, el f́tem estaba refexido a la interacción entre imanes, concepto dominado por la práctica totalidad de los alumnos; sin embargo, en el ítem correspondiente del test posterior, no sólo se pretendía medir si conocían o no cómo interaccionaban dos solenoides, sino que previamente debían saber asignarles extremos Sur y Norte en función del sentido de la corriente a través de ellos.

En el gráfico 2, la barra negra muestra el porcentaje de alumnos que han resuelto correctamente el ítem correspondiente del test posterior. La barra blanca alude al test de MLP.

\section{Gráfico 2}

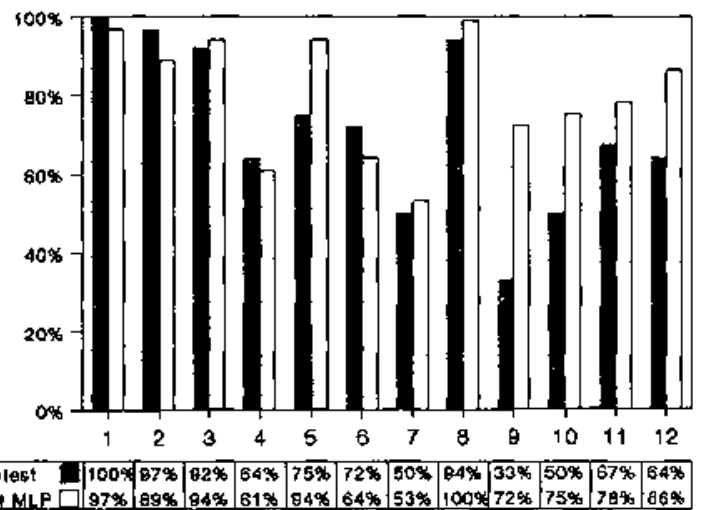

La comparación entre ambos tests, el posterior y el de MLP, también arroja diferencias -aunque ahora no significativas-, pero en sentido contrario al que preveíamos que pudieran existir. El cuadro II resume este hecho.

Cuadro II

Valor medio de ítems acertados en el test posterior

Valor medio de items acertados en el test de MLP $80 \%$

Incremento absoluto

Un análisis pormenorizado muestra que los ítems $1,2,4$ y 6 manifiestan pequeños retrocesos no significativos estadísticamente, que pueden ser perfectamente interpretados como procesos aislados de carácter obliterativo. Sin embargo, en los demás se presenta ganancia. Esta no es relevante en los ítems 3,7 y 8 , pero sí consideramos que lo es en el resto (ítems $5,9,10,11$ y 12), lo cual determina ese incremento final del $8 \%$, no significativo (coeficiente F de Sheffe 1.224).

El hecho de que en una buena parte de los ítems aumente per se la cantidad de conocimientos con el transcurso del tiempo, en lugar de producirse olvido, choca con las previsiones. Sólo si suponemos que durante ese lapso de tiempo ha habido obtención por parte del alumno de nueva información -o revisión de la disponible hasta entonces-, podremos dar explicación del incremento que se produce en los mencionados ítems. Pudimos descubrir que, tras el test posterior, fue comentada la resolución a los ítems del mismo, discutiendo cuál era la solución correcta en cada caso, y por qué. Se había producido, por tanto, un proceso de adiestramiento posterior a la SEA.

Sólo los últimos ítems, relativos a objetivos de profundización, se ven afectados por este proceso de instrucción posterior. La mayor parte đe los primeros ítems, al haber sido resueltos correctamente casi por la totalidad de los alumnos, no fueron discutidos, por lo que permiten observar esos pequeños decrementos reveladores de procesos obliterativos. Al ser estas diferencias en su mayoría muy pequeñas, podemos seguir suponiendo que los conocimientos adquiridos durante la SEA -independientemente de los añadidos después de la mismapermanecen a largo plazo, anclados a las estructuras conceptuales de los alumnos. De todos modos, se hace necesaria una nueva experiencia para contrastar nuestra hipótesis de trabajo (Hipótesis 1.2).

En relación con la hipótesis 1.1, para realizar un análisis diferencial del rendiniento en función de la situación de partida, clasificamos las pruebas cumplimentadas por los alumnos formando dos grupos en función de los resultados del test previo. De esta forma conseguimos un primer grupo integrado por aquellos alumnos que sólo habian resuelto correctamente de 1 a 3 ítems en el test previo (el $53 \%$ del total), y un segundo grupo formado por aquellos alumnos que habían respondido adecuada- 
mente más de 3 ítems en el mismo test (eI $47 \%$ restante). El estudio comparado del rendimiento de estos dos grupos reveló el efecto esperado. Los siguientes cuadros con sus correspondientes gráficos nos permiten comparar los resuitados de ambos grupos. Los porcentajes que en ellos aparecen representan los valores promedio de ítems acertados en cada test.

\section{A. NIVEL DE MÍNIMOS (ÍTEMS 1-7)}

Cuadro Ill

\begin{tabular}{llllll}
\hline & $\begin{array}{l}\text { Test } \\
\text { previo }\end{array}$ & $\begin{array}{l}\text { Test } \\
\text { posterior }\end{array}$ & $\begin{array}{l}\text { Incremento } \\
\text { parcial }\end{array}$ & $\begin{array}{l}\text { Test } \\
\text { de MLP }\end{array}$ & $\begin{array}{l}\text { Incremento } \\
\text { final }\end{array}$ \\
Gr. 1 & $38 \%$ & $80 \%$ & $42 \%$ & $77 \%$ & $39 \%$ \\
Gr. 2 & $66 \%$ & $76 \%$ & $10 \%$ & $79 \%$ & $13 \%$ \\
\hline
\end{tabular}

Gráfico 3

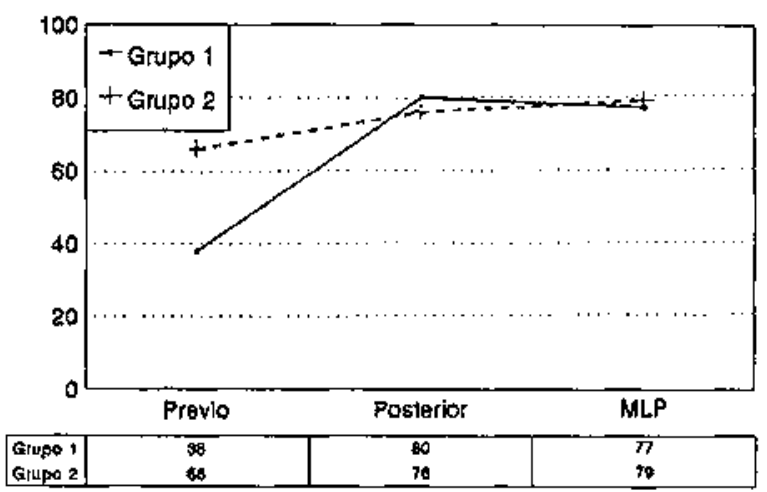

Como se aprecia, el incremento en el aprendizaje del grupo 2-los alumnos, en teoría, mejor preparados inicialmente- es un $13 \%$ frente a un $39 \%$ logrado por el grupo 1 -alumnos con peor conocimiento previo-. Ello supone un efecto de homogeneización en el dominio final de los dos grupos, a pesar de la diferencia en el dominio previo ( $38 \%$ y $66 \%$ sobre los 7 f́tems de mínimos). El efecto se acentúa en el caso del test de MLP en donde los resultados son del $77 \%$ y $79 \%$ respectivamente (siempre respecto a los 7 ítems de mínimos). Por otro lado, en cuanto a mínimos, no hay variación en el dominio medio entre los test posterior y MLP, que queda fijado en un $78 \%$.

\section{B. NIVEL DE PROFUNDIZACIÓN (ÍTEMS 8-12)}

\begin{tabular}{llll} 
& \multicolumn{4}{c}{ Cuadro IV } \\
\hline & $\begin{array}{l}\text { Test } \\
\text { posterior }\end{array}$ & $\begin{array}{l}\text { Test } \\
\text { MLP }\end{array}$ & Incremento \\
Gr. 1 & $64 \%$ & $83 \%$ & $19 \%$ \\
Gr. 2 & $59 \%$ & $80 \%$ & $21 \%$ \\
\hline
\end{tabular}

Grafico 4

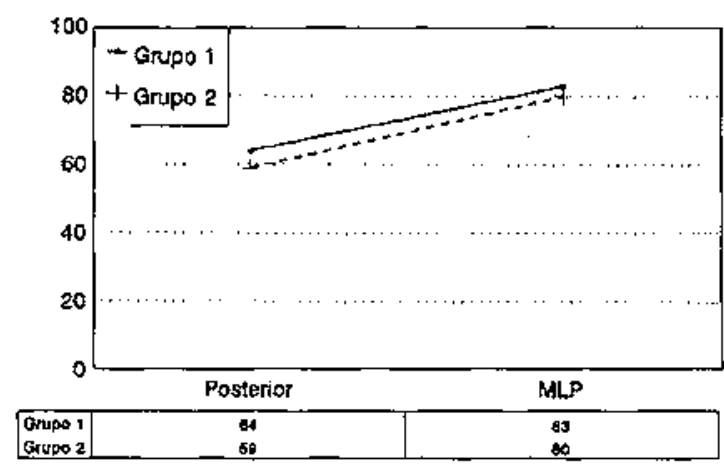

En este nivel se percibe, de nuevo, el efecto de homogeneización entre ambos grupos en el test posterior $(64 \%$, $59 \%)$, acentuándose a largo plazo $(83 \%, 80 \%)$. En este último caso, sin embargo, el rendimiento medio de ambos grupos se ha visto incrementado de un $62 \%$, en el test posterior, a un $80 \%$ en el test de MLP, lo que confirma el hecho de que el ya comentado proceso involuntario de adiestramiento posterior a la SEA se ha dado principalmente en los objetivos de profundización.

\section{RESULTADOS GLOBALES (ÍTEMS 1-12.) $(12$ aciertos $=100 \%)$}

Si consideramos el rendimiento de ambos grupos desde el punto de vista de mínimos y de profundización conjuntamente, el efecto de homogeneización queda aún más patente.

Cuadro V

\begin{tabular}{llllll}
\hline & $\begin{array}{l}\text { Test } \\
\text { previo }\end{array}$ & $\begin{array}{l}\text { Test } \\
\text { posterior }\end{array}$ & $\begin{array}{l}\text { Incremento } \\
\text { parcial }\end{array}$ & $\begin{array}{l}\text { Test } \\
\text { de MLP }\end{array}$ & $\begin{array}{l}\text { Incremento } \\
\text { final }\end{array}$ \\
Gt. 1 & $22 \%$ & $74 \%$ & $52 \%$ & $80 \%$ & $58 \%$ \\
Gr. 2 & $39 \%$ & $69 \%$ & $30 \%$ & $80 \%$ & $41 \%$ \\
\hline
\end{tabular}

Gráfico 5

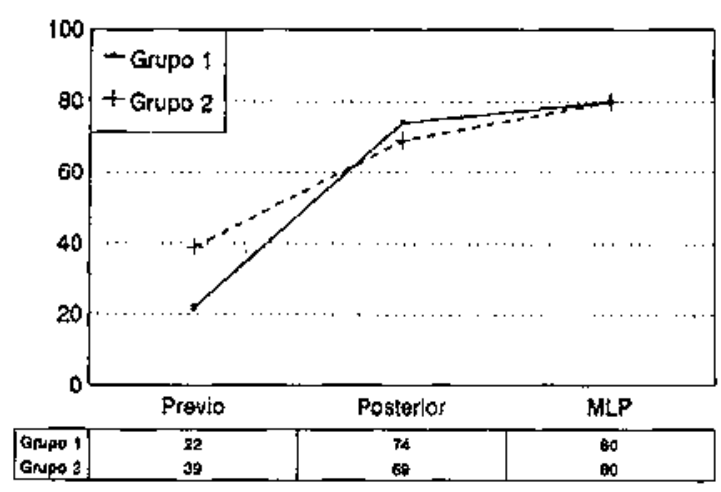

ENSEÑANZA DE LAS CIENCIAS, 1993, 11 (3) 
Aunque la situación de partida fuera dispar ( $22 \%$ y $39 \%$ respectivamente), la situación última es idéntica para ambos grupos (un $80 \%$ ). Y esto se ha producido a pesar de haber previsto que aquellos alumnos con más conocimientos íniciales del tema pudieran seguir desarrollándose en su nivel, mediante actividades de mayor dificultad. Por supuesto, todos los ejercicios-sencillos y difícilesestaban a disposición de todos los alumnos. Por tanto, aquéllos peor preparados previamente han debido poseer la suficiente motivación para realizar un esfuerzo suplementario que les permitiera equipararse con sus compañeros inicialmente más conocedores del tema.

Desde un punto de vista ausubeliano, también puede ser explicado este efecto de homogeneización al considerar que los inclusores que se precisan para este tema y su facilidad de obtención han podido determinar que aqueIlos alumnos con cierta desventaja inicial alcancen rápidamente un nivel aceptable de comprensión desde el inicio de la SEA. Esto viene corroborado por el hecho de que la homogeneización no sólo se ha producido a nivel de contenidos mínimos, como preveíamos, sino también a niveles de profundización, en donde esperábamos encontrar ya alguna diferencia entre ambos grupos. Creemos que elio ha sido factible gracias a que en Ia elaboración del diseño instruccional se ha partido, no desde conocimientos que los alumnos debían tener de cursos anteriores, sino desde conceptos mucho más primigenios presentes en la práctica totalidad de los sujetos. Ello ha provocado que rápidamente ambos grupos hayan igualado su rendimiento en las primeras fases de la SEA, permitiendo un avance simultáneo en el dominio de los contenidos. Esto parece confirmar nuestra hipótesis 1.1.

\section{CONCLUSIONES}

Los objetivos principales de nuestra investigación (comprobar experimentalmente la aplicabilidad del modelo de aprendizaje de Ausubel en la SEA, a través de la preparación y puesta a punto de los instrumentos experimentales necesarios, y la confección del diseño instruccional correspondiente) han sido, en nuestra opinión, satisfactoriamente alcanzados.

Las hipótesis formuladas y abordadas en este trabajo presentan un nivel de verificación que consideramos adecuado, probando así la eficacia potencial de diseños instruccionales ausubelianos -en los cuales se han tenido en cuenta los procesos psicológicos de construcción del conocimiento- en el tratamiento de contenidos científicos que se representen mediante constructos poco elaborados y poco arraigados previamente en las estructuras conceptuales de los aprendices.

EI efecto de homogeneización observado nos permite pensar que la elección de inclusores es satisfactoria en principio, que el organizador previo de nuestro diseño instruccional puede ser adecuado y que el tratamiento y la organización del contenido han sido suficientemente elaborados para los propósitos perseguidos.
Nos resta ahora seguir reflexionando sobre el modo de perfeccionar aún más nuestro diseño instruccional para lograr mejores resultados, así como el introducir las mejoras oportunas en el material didáctico y experimental.

Lo realizado hasta ahora es sólo el inicio de un proyecto a más largo plazo centrado en la didáctica de la física en niveles de secundaria, en el cual pretendemos ampliar la investigación a una muestra más extensa, contando con la colaboración de varios centros con sus correspondientes profesores, y comparando además nuestro modelo de instrucción con otras situaciones educativas de control, cuyos diseños instruccionales posean una estructuración lógica de los contenidos (similar a la de los textos), aunque ajena a Ios procesos psicológicos del aprendizaje.

Por otra parte, hemos observado un incremento de la motivación en el transcurso de nuestra investigación. Por ello, estamos investigando si existe o no un incremento medible en variables motivaciones asociadas con este diseño, y su posible correlación con el rendimiento de los alumnos. Sabemos que la motivación activa la comprensión. Recíprocamente, creemos que, activando la comprensión a partir de una secuencialización adecuada del contenido, pueden activarse asimismo estrategias de apoyo, como la motivación. Nos basamos en una de las consecuencias lógicas que pueden derivarse del modelo: un diseño instruccional ausubeliano en el que el orden de presentación de los contenidos sea compatible con los procesos psicológicos del aprendizaje debe producir un aumento en la motivación como resultado de la asimilación cómoda -desde el punto de vista psicológico- de los conocimientos.

En otro orden de cosas, Ia variable tiempo a menudo marca diferencias entre unas y otras metodologías en el aula. Dependiendo de cuáles sean los planteamientos didácticos básicos, esta variable será más o menos relevante. En los niveles superiores (COU, universidad), modelos similares a «el alumno como investigador principiante», desarrollado, entre otros, por Gil y sus colaboradores (Gil et al. 1991, González et al. 1992, Salinas 1992) se revelan como idóneos -al menos hasta donde Ia investigación en curso demuestra-, ya que el rol de científico es asumido con facilidad por parte de los aprendices. En este modelo, la variable tiempo es irrelevante, puesto que en la investigación científica habitual, no se conoce qué tiempo se requiere para alcanzar el objetivo. En los niveles inferiores, sin embargo, se presentan otras dificultades que vienen de la no asunción de este rol, la diversidad de materias, las ligaduras y exigencias legales, etc.

Nosotros asumimos que el sistema educativo (al menos los niveles inferiores) pretende, del estudiante, no sólo la consecución de una cierta cantidad de objetivos, sino que éstos sean alcanzados en un período de tiempo determinado. Esta limitación temporal determina en la práctica que una metodología será útil si logra buenos resultados en comprensión - a corto y largo plazo- y en motivación, pero además en un intervalo de tiempo programado. 
Pretendemos pues controlar, en nuestra investigación en curso, la variable tiempo, estudiando si nuestro diseño permite un mayor ritmo en el aprendizaje significativo, comparando los resultados de dominio en función del tiempo con los de los grupos control.
En caso de confirmarse las hipótesis de nuestro trabajo, ello puede significar haber avanzado en la definición de los limites de validez de un modelo asequible al cuerpo docente que imparte disciplinas científicas, de una metodología constructivista suficientemente testada compatible con los propósitos de la Reforma.

\section{REFERENCIAS BIBLIOGRÁFICAS}

AUSUBEL, D.P., 1976. Psicología educativa: un punto de vista cognitivo. (Trillas: México).

CLOUGH, E.E. y DRIVER, R., 1986. A study consistency in the use of students'conceptual frameworks across different task context, Science Education, 70 (4), pp. 373-496.

COLL, C., 1987. Psicología y currículum. (Laia: Barcelona).

COLL, C., 1991. Concepción constructivista y planteamiento curricular, Cuadernos de Pedagogía, 188, pp. 8-11.

CUBERO, R., 1989. Cómo trabajar con las ideas de los alumnos. (Díada: Sevilla).

DRIVER, R. y ERICKSON, G., 1983. Theories-in-action: some theoretical and empirical issues in the study of students' conceptual frameworks in science, Studies in Science Education, 10 , pp. 37-60.

DRIVER, R., GUESNE E. y TIBERGHIEN, A., 1989. Ideas cientificas en la infancia y la adolescencia. (Morata: Madrid).

EDWARDS, D. y MERCER, N., 1988. El conocimiento compartido. El desarrollo de la comprensión enelaula.(Paidós, MEC: Madrid).

FORTES, M.C., 1991. Tesis doctoral. Valencia.

GIL, D., 1983. Tres paradigmas básicos en la enseñanza de las ciencias, Enseñanza de las ciencias, 1, p. 26.

GIL, D., 1986. La metodología científica y la enseñanza de las ciencias: unas relaciones controvertidas, Enseñanza de las Ciencias, 4, p. 111.

GIL, D., CARRASCOSA, J., FURIO, C. y MARTÍNEZTORREGROSA, J., 1991, La enseñanza de las ciencias en la educación secundaria. (HORSORI- ICE de la Universitat de Barcelona: Barcelona).

GILBERT, J.K. y WATTS, D.M., 1983. Concepts, misconceptions and alternative conceptions: changing perspectives in science education, Studies in Science Education, 10, pp. 61-98.

GONZÁLEZ, E., 1992. Tesis de master. Universitat de València.
KELLY,G., 1955.The psychologyof personal constructs. (Norton: Nueva York).

LANGFORD, P., 1989. El desarrollodel pensamiento conceptual en la escuela. (Paidós: Barcelona).

LATORRE, A., 1990. Psicología del proceso de enseñanzal aprendizaje: La situación educativa. (Nau Llibres: Valencia).

NOVAK, J.D., 1979, The perception learning paradigm, Journal of Research in Science Teaching, 16, p. 481 .

NOVAK, J.D., 1986. The importance of emerging constructivist epistemology for mathematics education, Journal of Mathematical Behaviour, 5, pp. 181-184.

NOVAK, J.D., 1987. Human constructivism: Towards a unity of psychological and epistemological meaning making, en: Porlán, R., García, J.E. y Cañal, P. (compiladores), 1988, Constructivismo yenseñanza de las cienciass. (Diada: Sevilla).

NOVAK, J.D. y GOWIN, D.B., 1988. Aprendiendo a aprender. (Martinez-Roca: Barcelona).

OSBORNE, R.J. y GILBERT, J.K., 1980. A technique for exploring the student's view of the world, Physics Education, 15, pp. 376-379.

OSBORNE, R.I. y WITTROCK, M.C., 1983. Learning science: A generative process, Science Education, 67 (4), pp. 489-508.

SALINAS, J,, 1992. Tesis de tercer ciclo. Universitat de València.

SALTIEL, E. y VIENNOT, L., 1985. ¿Qué aprendemos de las semejanzas entre las ideas historicas y el razonamiento espontáneo de los estudiantes?, Enseñanza de las Ciencias, $3(2)$, pp. 137-144.

STRIKE,K. y POSNER, G., 1982. Conceptual change and science teaching, European Journal of Science Education, 4 (3), pp. $231-240$.

POSNER, G., 1982. Acommodation of a scientific conception: towards a theory of conceptual change, Science Education, 66 , pp. $7-10$. 
ANEXO I

OBJETIVOS

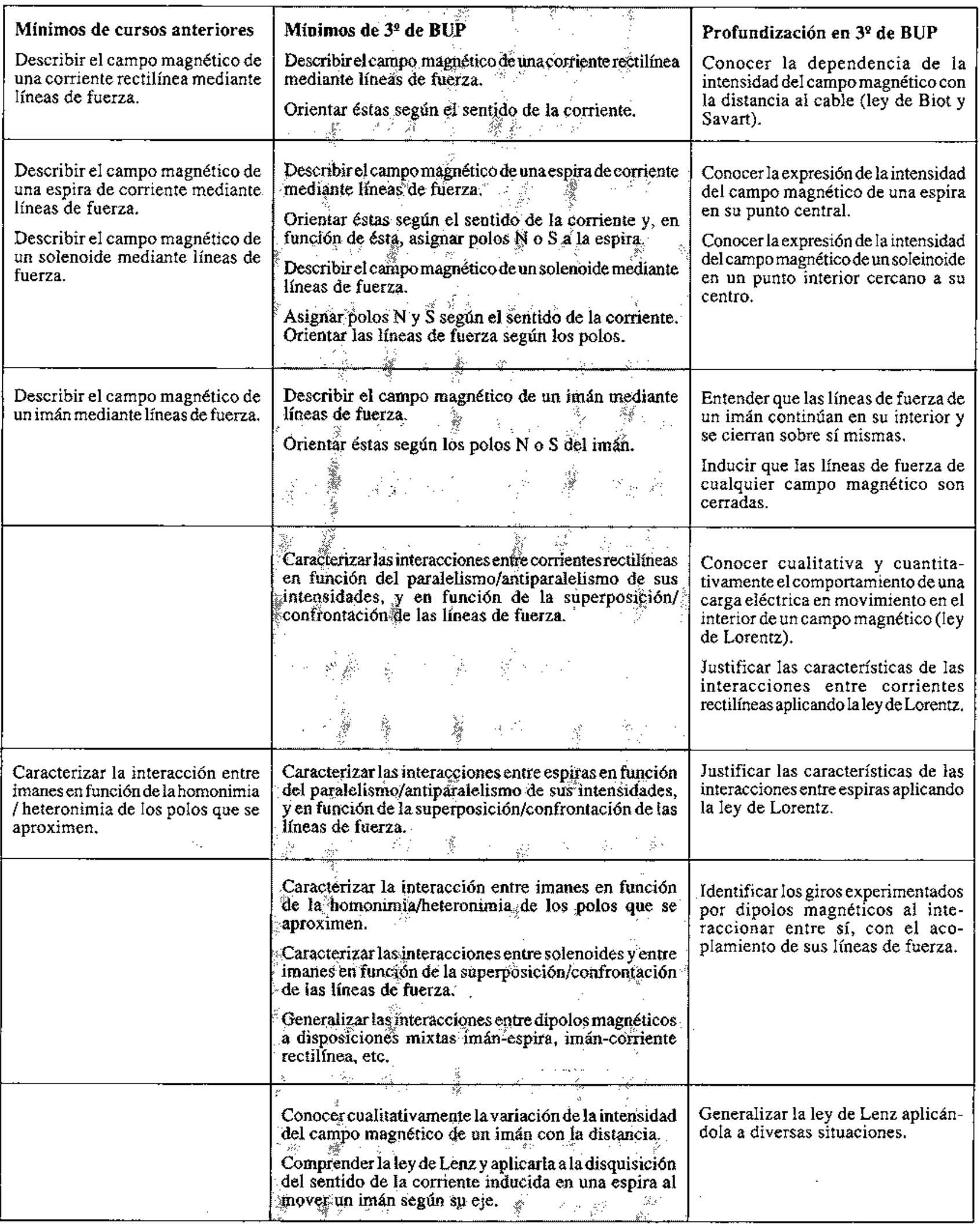




\section{ANEXO II. A \\ TEST PREVIO}

1. De los siguientes dibujos, ¿cuál describe correctamente las líneas de fuerza del campo magnético de un imán?

a)

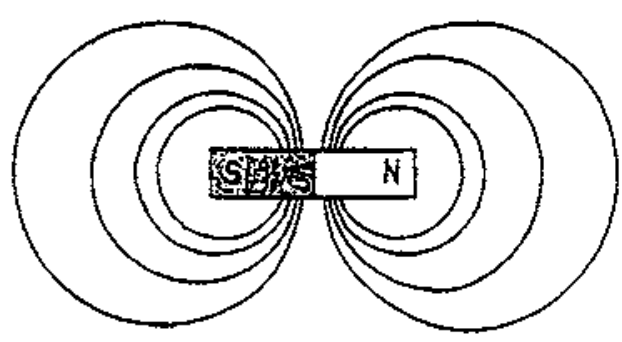

c)

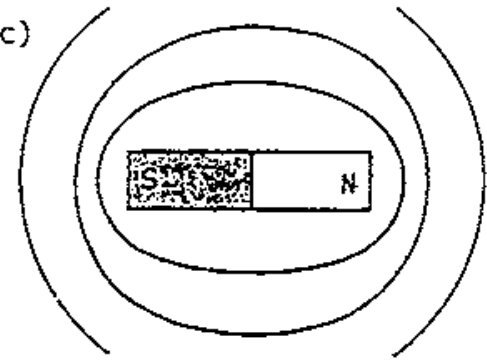

b)

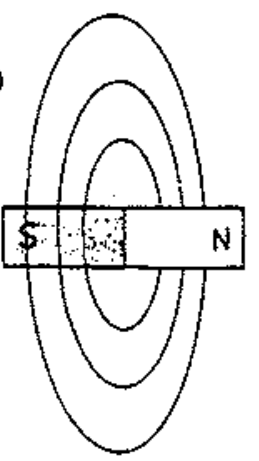

d)

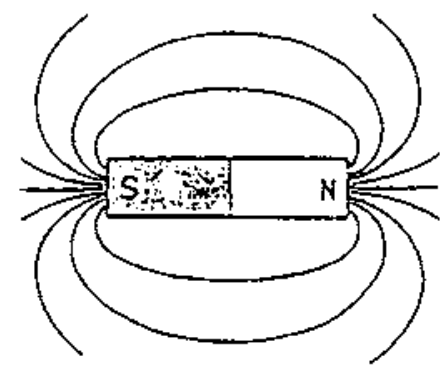

2. De los siguientes dibujos, ¿cuál describe correctamente las líneas de fuerza del campo magnético de una corriente continua rectilínea de intensidad I?

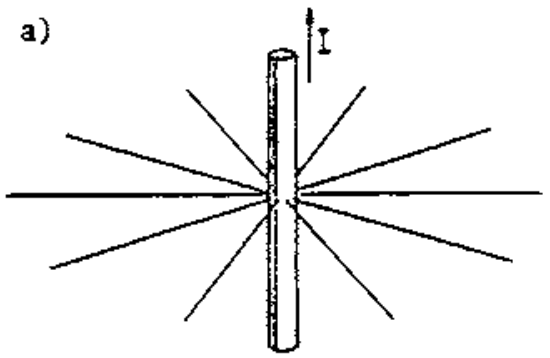

b)

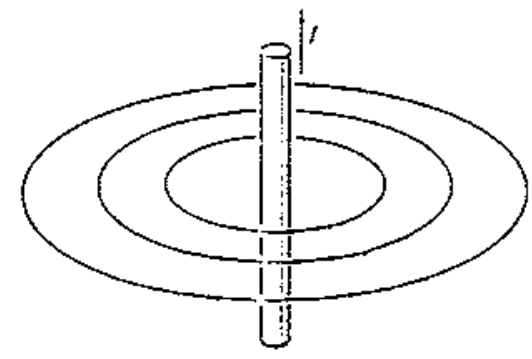

c) $\$$

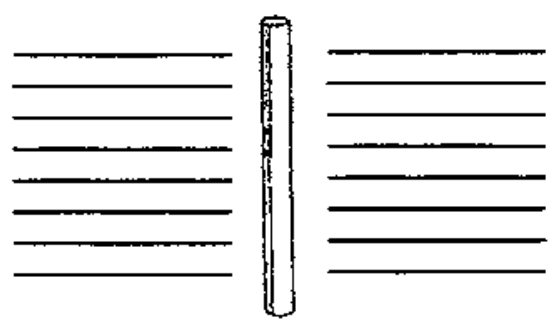

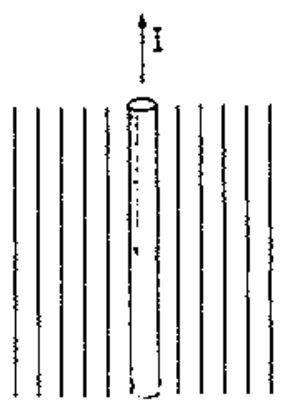




\section{ANEXO II. B}

\section{TEST POSTERIOR}

1. De los siguientes dibujos, ¿cuál describe correctamente las líneas de fuerza del campo magnético de un imán?
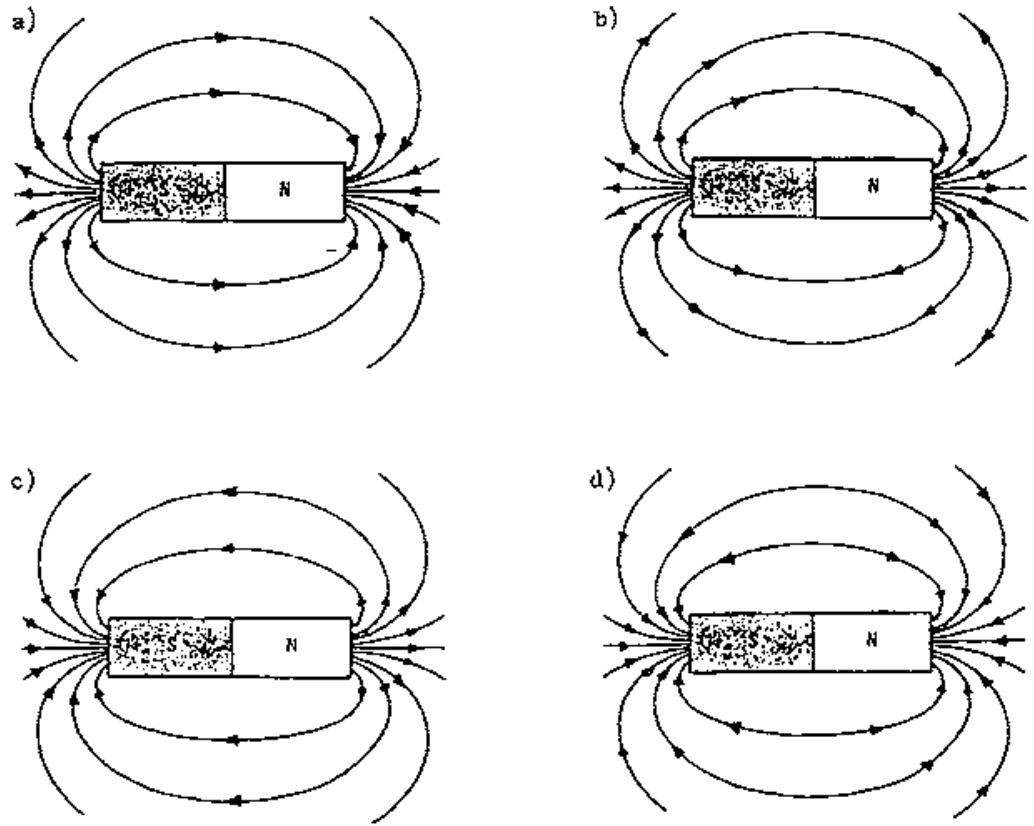

2. De los siguientes dibujos, ¿cuál describe correctamente las líneas de fuerza del campo magnético de una corriente continua de intensidad $\mathrm{I}$ ?
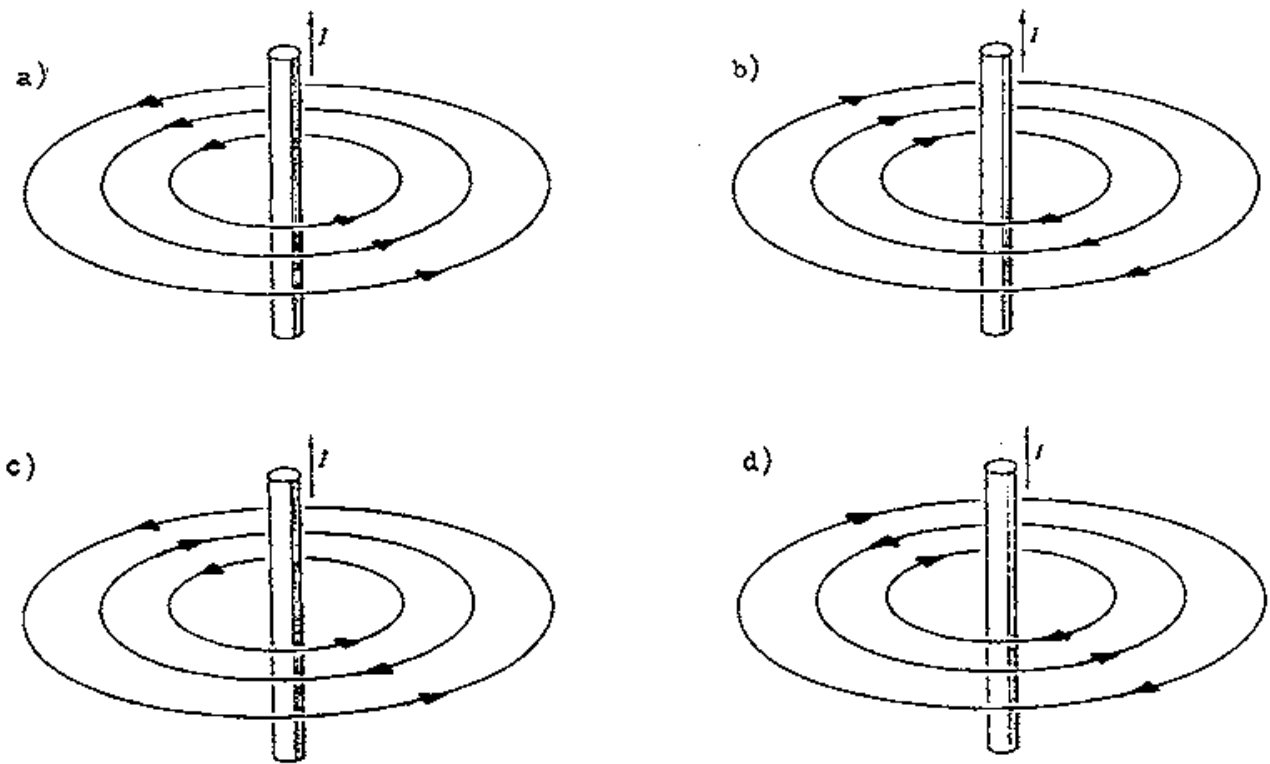


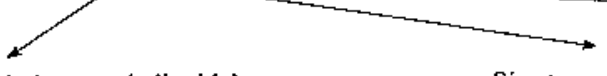

Si este movimiento es helicoidal, tenemos un

i este movimiento es circular, tenemos una

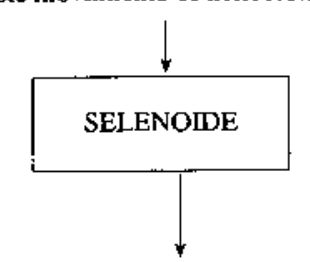

cuyo campo magnético se visualiza a través de las líneas de fuerza

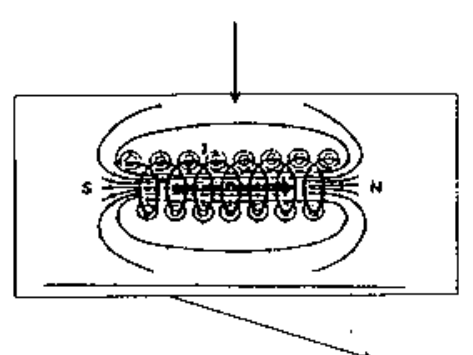

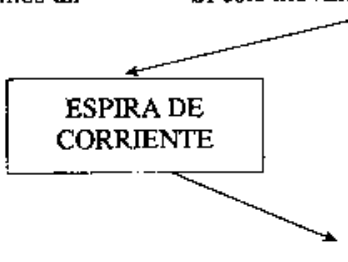

cuyo campo magnético se visualiza a través de las líneas de fuerza

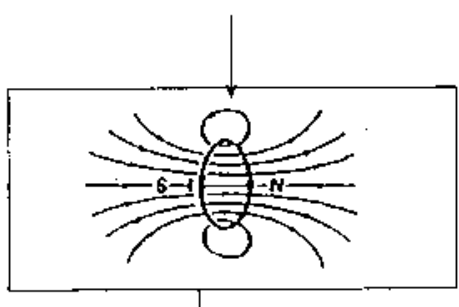

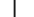

en los materiales ferromagnéticos la uperposicion de multitud de microespiras

(órbitas electrónicas) determina un

Si este movimiento es rectilíneo, tenemos una

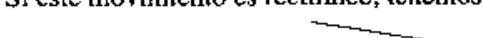

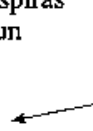

cuyo campo magnético se visualiza a través de las líneas de fuerza

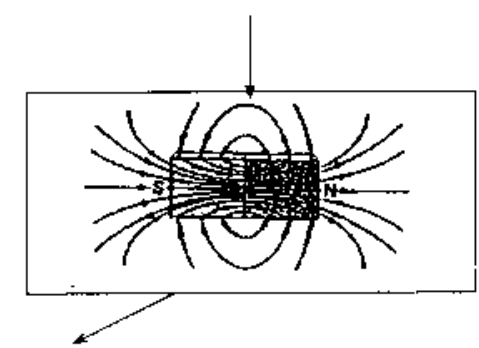

CORRIENTE RECTILÍNEA

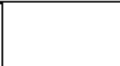

uyo campo magnético se visualiza través de las líneas de fuerza

$$
\checkmark
$$

Si se introduce en un campo magnético externo, tenderá a

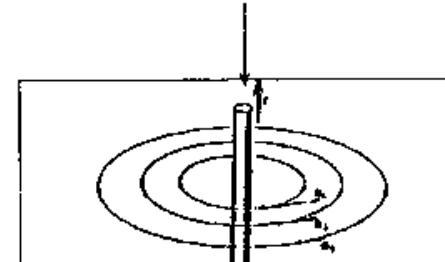

1

$\downarrow$ orientarse de modo que

as líneas de fuerza entran por su polo SUR y salen por el NORTE

las líneas de fuerza se supoerponen con las del campo exteno debido a que

dos imanes se atraen si se
encaran sus polos opuestos (sus
líneas de fuerza se superponen)
y se repelen si se encaran sus
polos iguales (sus líneas de
fuerza se confrontan)

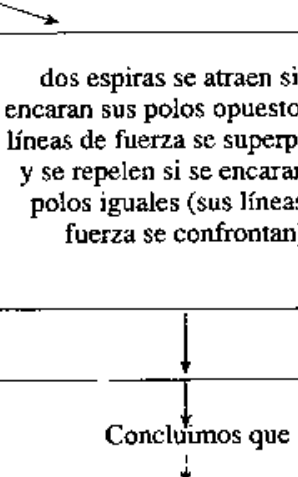

$n$

Conclumos que

\begin{abstract}
dos solenoides se atraen si se encarar sus polos opuestos (sus líneas de fuerza se superponen) y se repelen si se encaran sus polos iguales (sus líneas de fuerza se confrontan)
\end{abstract}

solenoides, imanes, espiras y

conrientes rectilíneas se atraen las líneas de fuerza de sus respectivos campos magnéticos se superponen y se repelen si las líneas de fuerza de sus campos magnéticos se confrontan
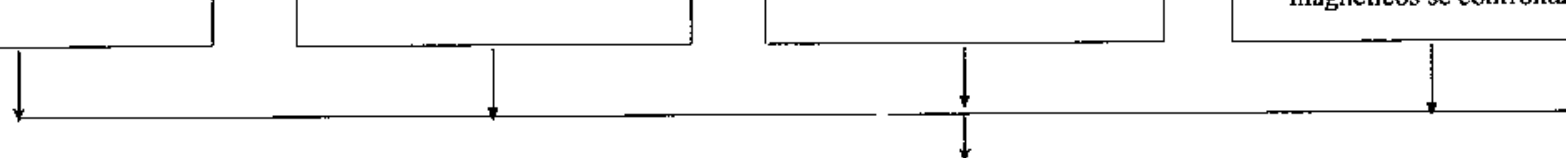